\title{
LLNL-TR-636200
}

\section{XNDL: METIS Partitioning Process}

L. E. Banks, P.D. Barnes, Jr., D.R. Jefferson April 2013

Technical Report

submitted to the

United States Army Research Lab (ARL) 


\section{Disclaimer}

This document was prepared as an account of work sponsored by an agency of the United States government. Neither the United States government nor Lawrence Livermore National Security, LLC, nor any of their employees makes any warranty, expressed or implied, or assumes any legal liability or responsibility for the accuracy, completeness, or usefulness of any information, apparatus, product, or process disclosed, or represents that its use would not infringe privately owned rights. Reference herein to any specific commercial product, process, or service by trade name, trademark, manufacturer, or otherwise does not necessarily constitute or imply its endorsement, recommendation, or favoring by the United States government or Lawrence Livermore National Security, LLC. The views and opinions of authors expressed herein do not necessarily state or reflect those of the United States government or Lawrence Livermore National Security, LLC, and shall not be used for advertising or product endorsement purposes.

This work performed under the auspices of the U.S. Department of Energy by Lawrence Livermore National Laboratory under Contract DE-AC52-07NA27344. 


\section{Table of Contents}

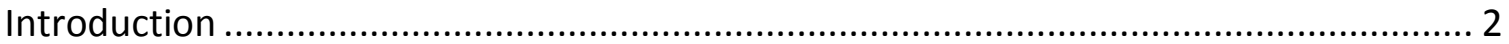

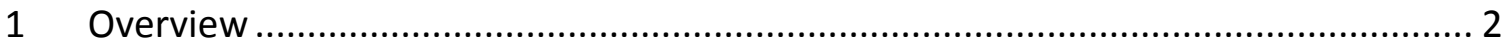

1.1 XNDL input specification with latency attributes .......................................... 2

2 Graph sectoring and reduction application ........................................................ 3

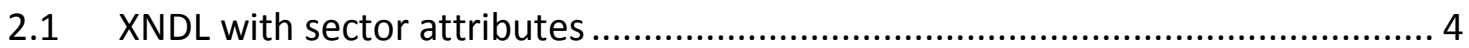

2.2 METIS input: reduced graph with edge weights .......................................... 4

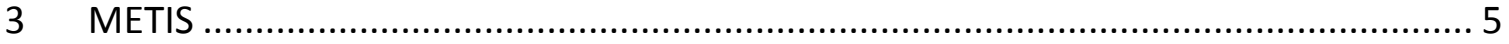

3.1 METIS Output: Mapping sector to MPI rank .............................................. 5

4 XSL Style Sheet: Add MPI attributes to XNDL …................................................ 5

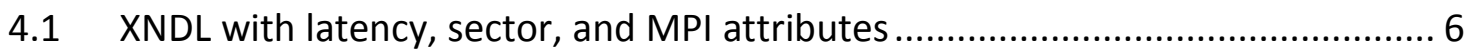

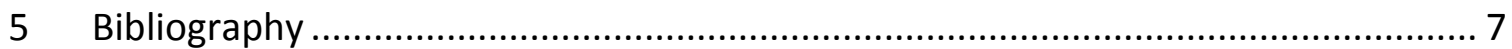

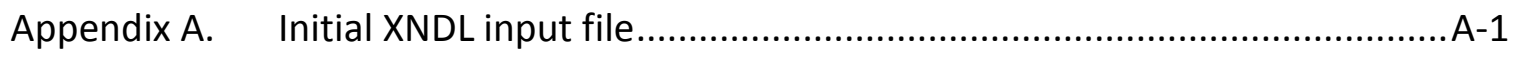

Appendix B. AddSystemld Python script ........................................................... 


\section{Introduction}

This document describes the partitioning process implemented within the XNDL framework in preparing ns-3 network simulation files for processing on parallel computing clusters at Lawrence Livermore National Laboratory (LLNL). The process employs the METIS graph partitioning tool in assigning network sectors to MPI ranks [1]. This work, conducted by LLNL, addresses a deliverable of the Statement of Work for the Proposed Research in Network Simulation specifically for the Army Research Laboratory under contract L145271.

\section{Overview}

In preparing a simulation input for a parallel run, the network topology must be partitioned and assigned to ranks for processing on the computing cluster. The process flow is shown in Figure 1.

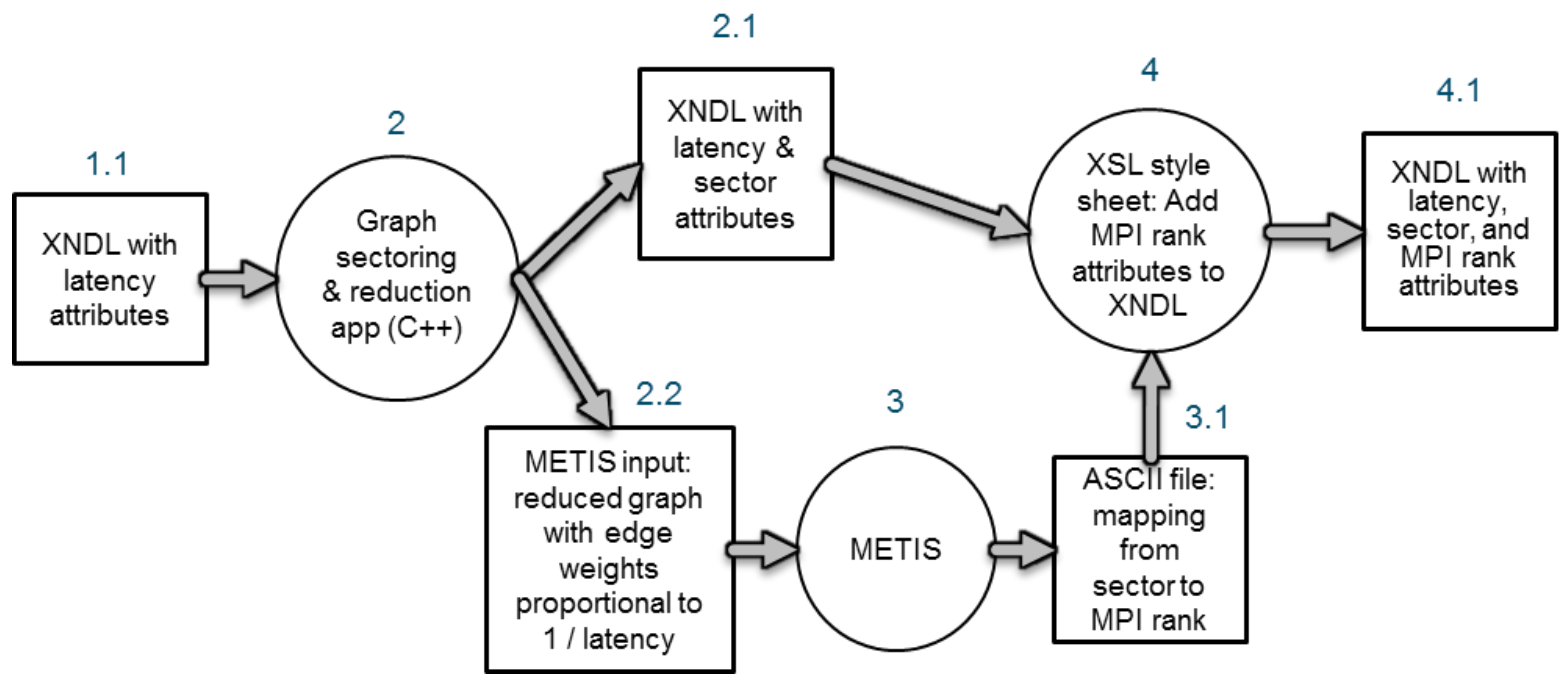

Figure 1: XNDL partitioning process flow. (Annotations correspond to topic sections in this paper.)

For illustration purposes we will assume that we are partitioning an XNDL model in the file model. xndl (included in Appendix A).

\subsection{XNDL input specification with latency attributes}

The network model is specified in the model . xndl file, with <NodeContainer $>$ elements defining the association between sets of nodes (see code Snippet 1: Initial XNDL input specification). These $<$ NodeContainer $>$ elements are referenced by $<$ subnet $>$ elements where the Delay parameter for the subnet is specified as well as each individual node 
Type. (This delay, or latency, will be used to calculate the edge weights for the graph input to METIS in the next iteration of the partitioning toolset.)

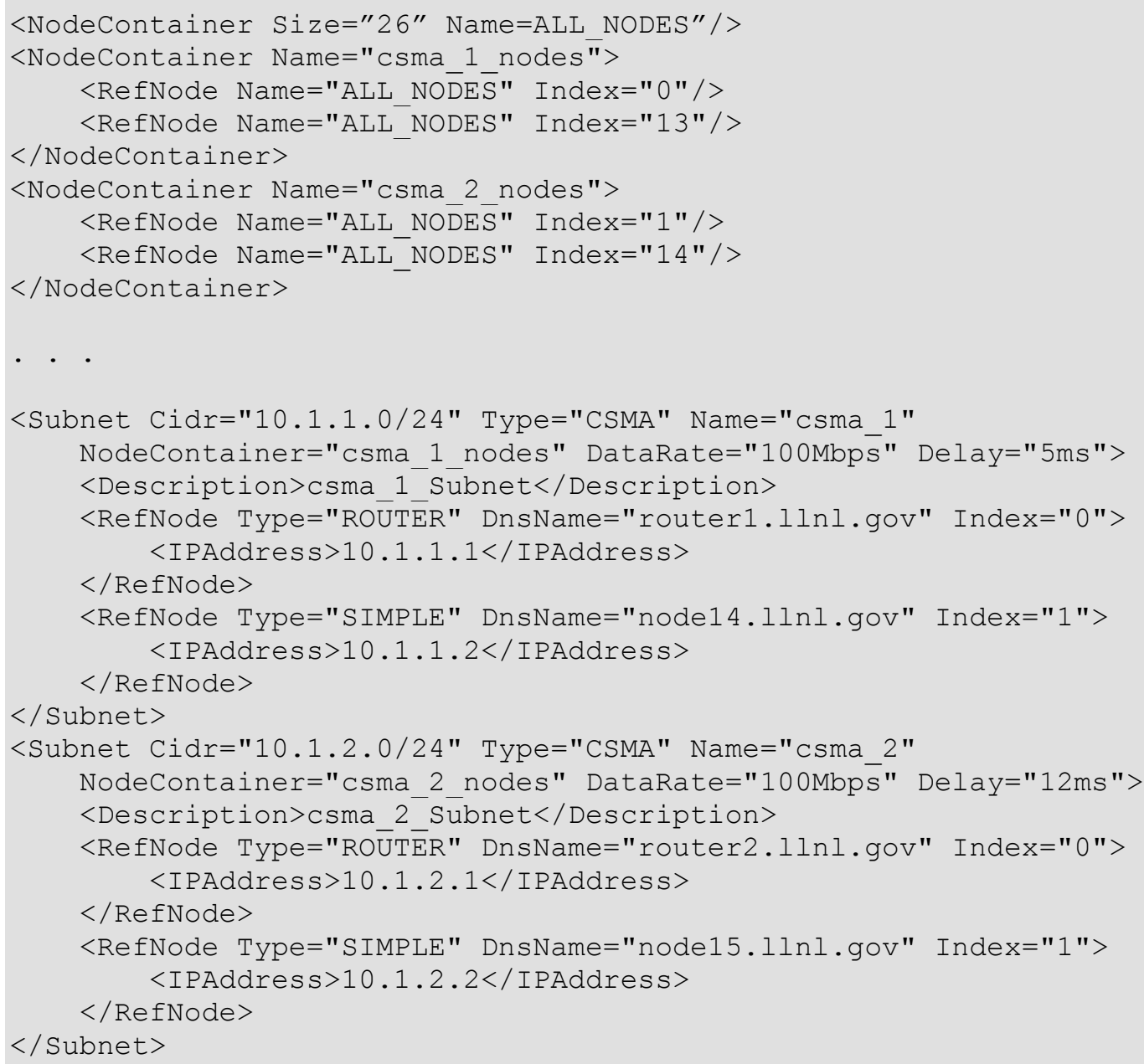

Snippet 1: Initial XNDL input specification

\section{Graph sectoring and reduction application}

The sector program is a stand-alone application that adds sector attributes to $<$ NodeContainers $>$ for which the associated set of nodes must reside on the same sector (e.g. cmsa nodes) and adds sector attributes to individual $<$ refnodes $>$ that can be placed in a separate sector from other nodes contained in their parent $<$ NodeContainer $>$ (e.g. P2P nodes). Logic exists to handle bridged csma channels, which 
should be placed in the same sector, as well as the handling of router-only nodes, which belong in independent sectors.

\subsection{XNDL with sector attributes}

The sector-annotated XNDL is output as model. sector. xndl (see Snippet 2: XNDL output from graph sectoring tool). as well as a sector graph in METIS input format.

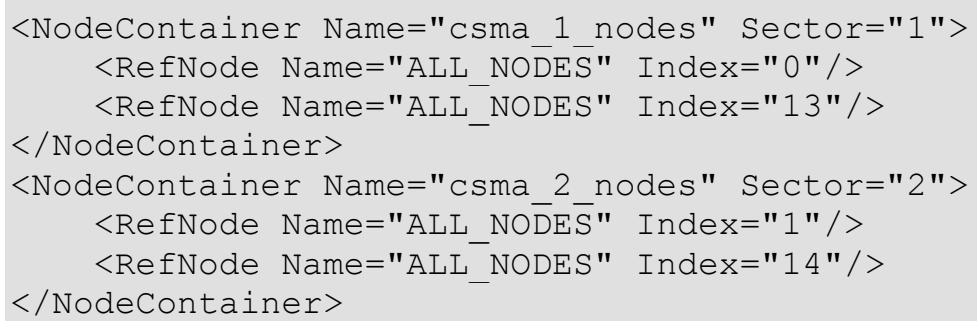

Snippet 2: XNDL output from graph sectoring tool

\subsection{METIS input: reduced graph with edge weights}

The graph sectoring tool also produces an input file for the METIS partitioning: model sector. metis (see Snippet 3: Graph sectoring tool output file for METIS input).

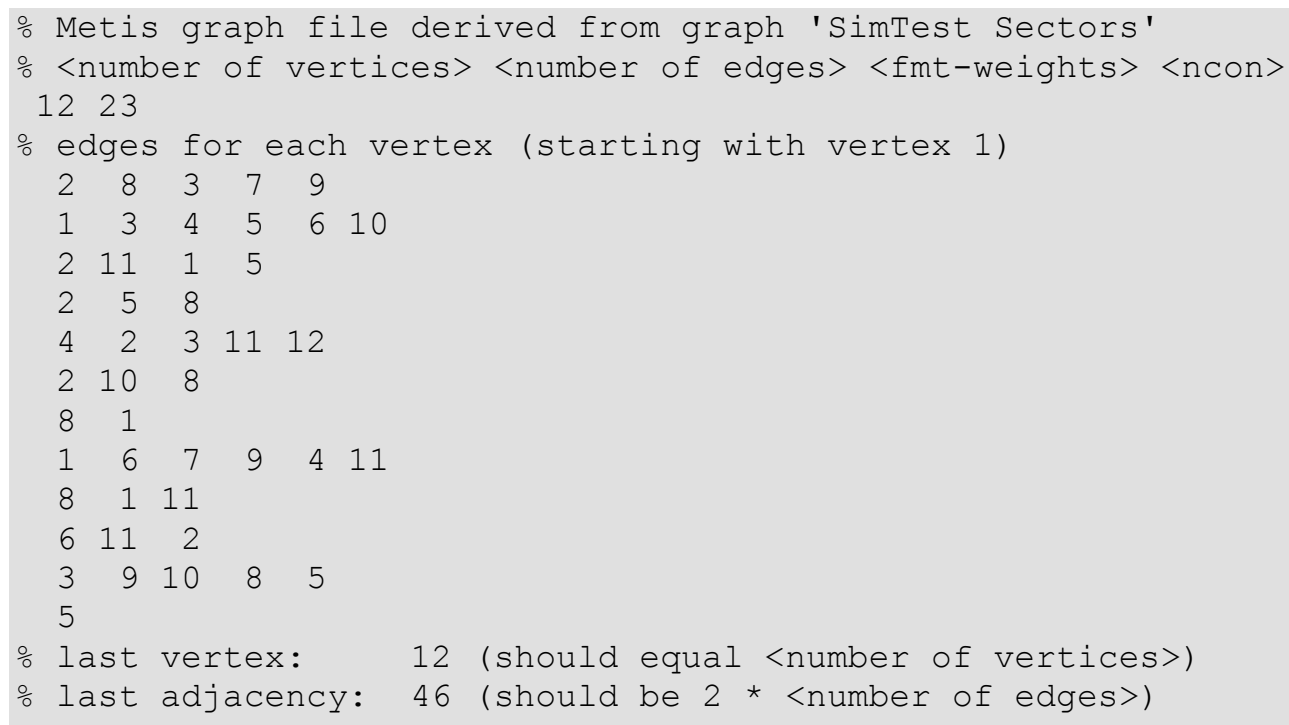

Snippet 3: Graph sectoring tool output file for METIS input 
The sector-labeled XML description file model. sector. xndl and the Metis input file model. sector.metis can be reused for different partitioning strategies, different numbers of MPI ranks, or different partitioning functions.

\section{METIS}

The METIS graph partitioning tool is used to assign sectors to MPI ranks based on its partitioning logic [1]. A typical command line to partition the model into 4 ranks would be:

$\$$ gpmetis model.sector.metis 4

\subsection{METIS Output: Mapping sector to MPI rank}

The output of gpmetis is written to model. sector.metis.part. 3 (see Snippet 4: METIS output file).

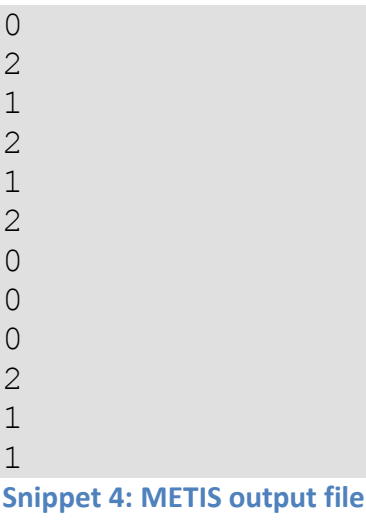

The sector number corresponds to the row in the output file. For instance, with this partitioning sector 1 will be assigned to rank 0 , sector 2 assigned to rank 2 , etc.

\section{XSL Style Sheet: Add MPI attributes to XNDL}

To produce the final simulation input specification file a python script, AddSystemId.py, takes as input: model. sector. xndl file, the XNDL with section attributes, an XSLT transform to apply the sector-to-rank assignments, and the METIS output file, model. sector. metis. part.3. In order for the XSLT transform to process the METIS output file it must first XMLize the file by simply adding XML tags to the rank entries. (See Snippet 5: XMLized METIS output file) 


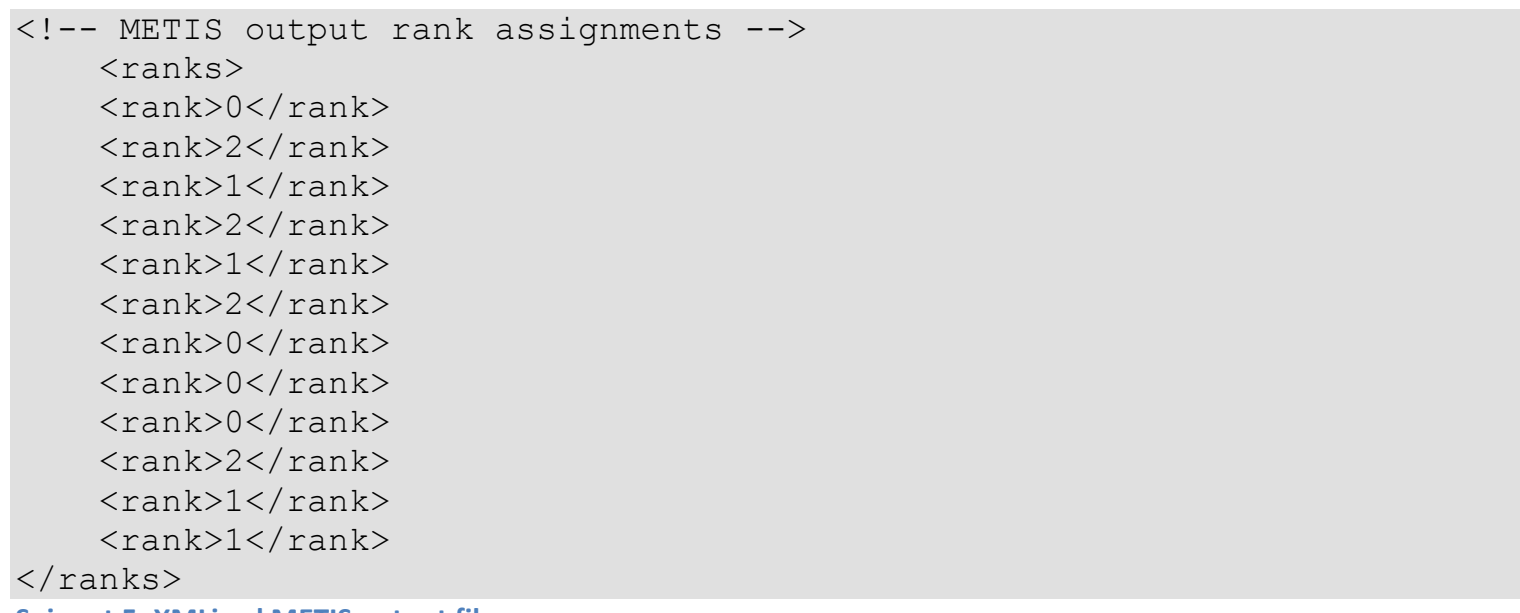

Snippet 5: XMLized METIS output file

The result of $X M L i z i n g$ the Metis output file is saved as model.sector.metis.part.3.xml.

The XSLT transform is then invoked to add the systemId attribute to the $<$ NodeContainers $>$ and/or $<$ RefNode $>$ elements of the XNDL file.

The syntax of the Python script is:

\$ python addSystemId.py -x [XNDL Sector file] -t [XSLT transform file] -m [METIS sector to rank map file]

\subsection{XNDL with latency, sector, and MPI attributes}

Output is written to stdout so can be redirected to create the final XNDL simulation input file, which would typically be saved as model.sector.metis.part.3.xndl. (See Snippet 6: Final simulation input file)

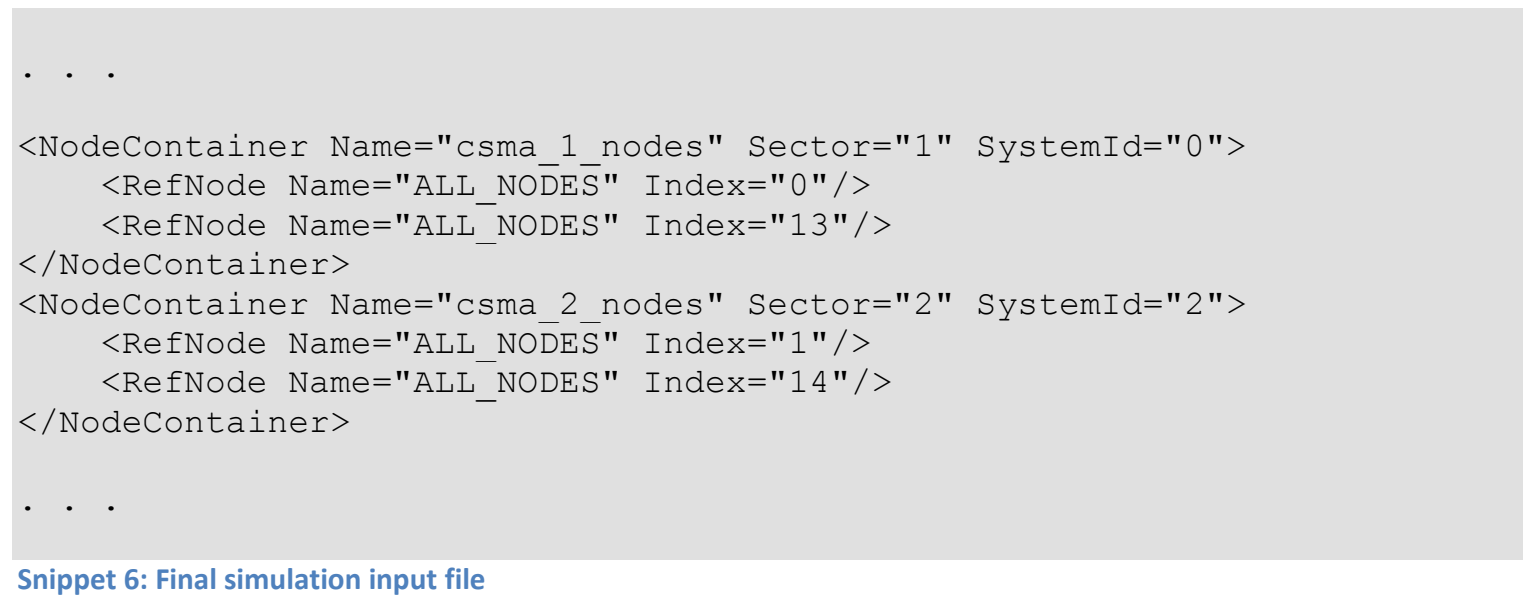

The final output XNDL is now fully annotated for a MPI-based simulation. 


\section{Bibliography}

[1] Karypis Lab, "METIS - Serial Graph Partitioning and Fill-reducing Matrix Ordering," 30 3 2013. [Online]. Available:

http://glaros.dtc.umn.edu/gkhome/metis/metis/overview. [Accessed 254 2013].

[2] L. Banks, P. Barnes, D. Jefferson and S. Nikolaev, "XML Network Description Lanaguage (XNDL) for ns-3," 2013. 
LLNL-TR-636200

\section{Appendix A. Initial XNDL input file}

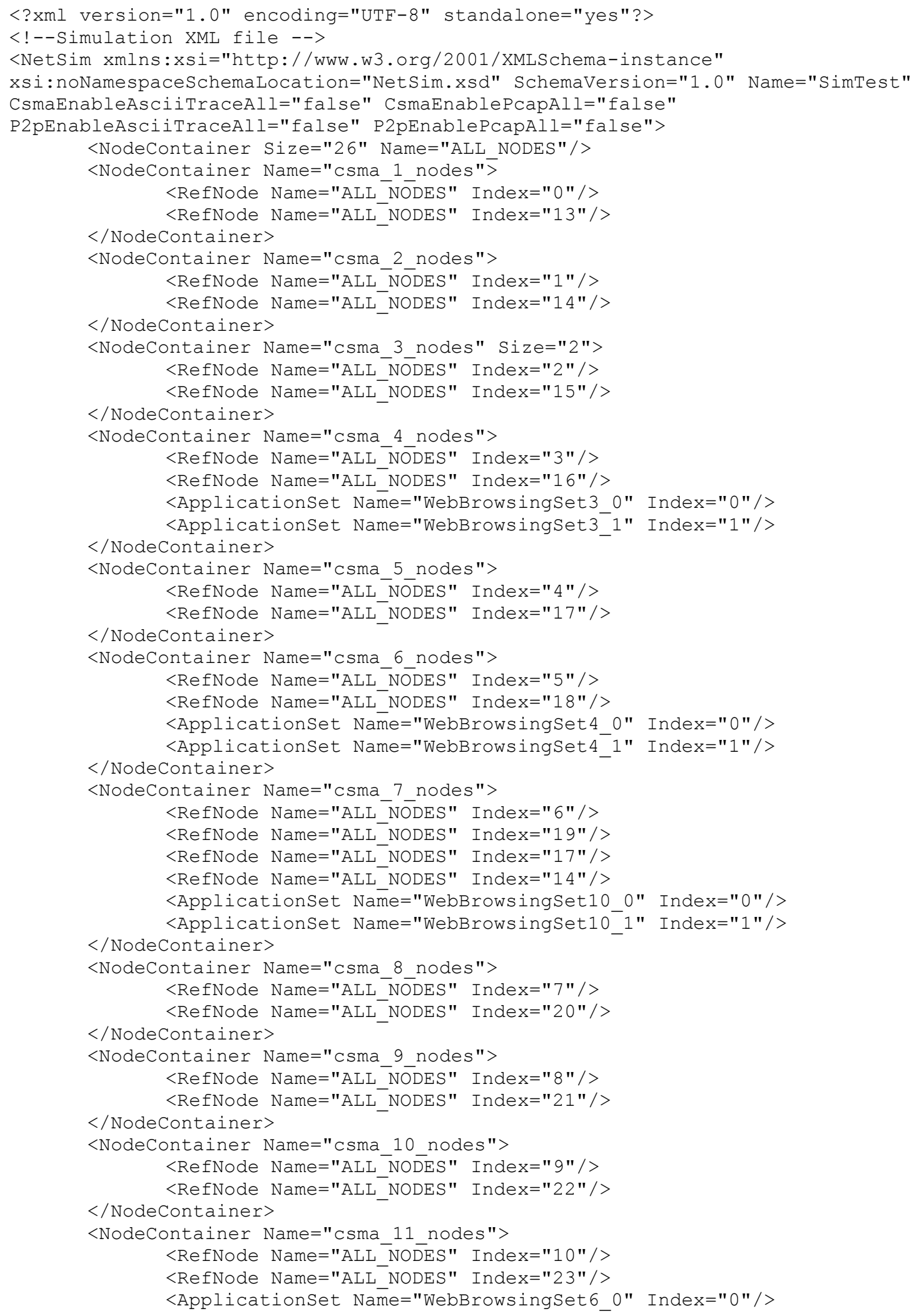




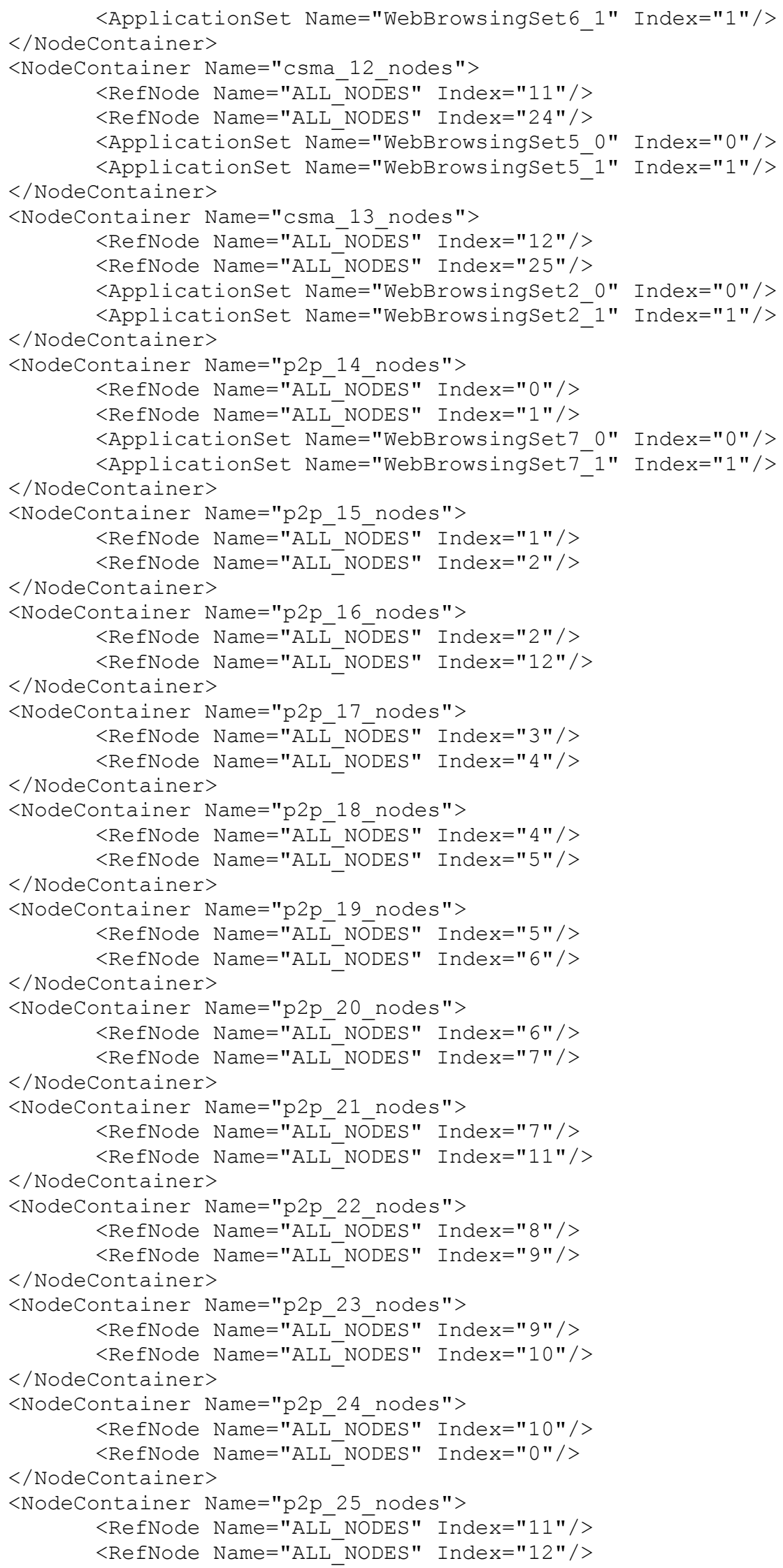




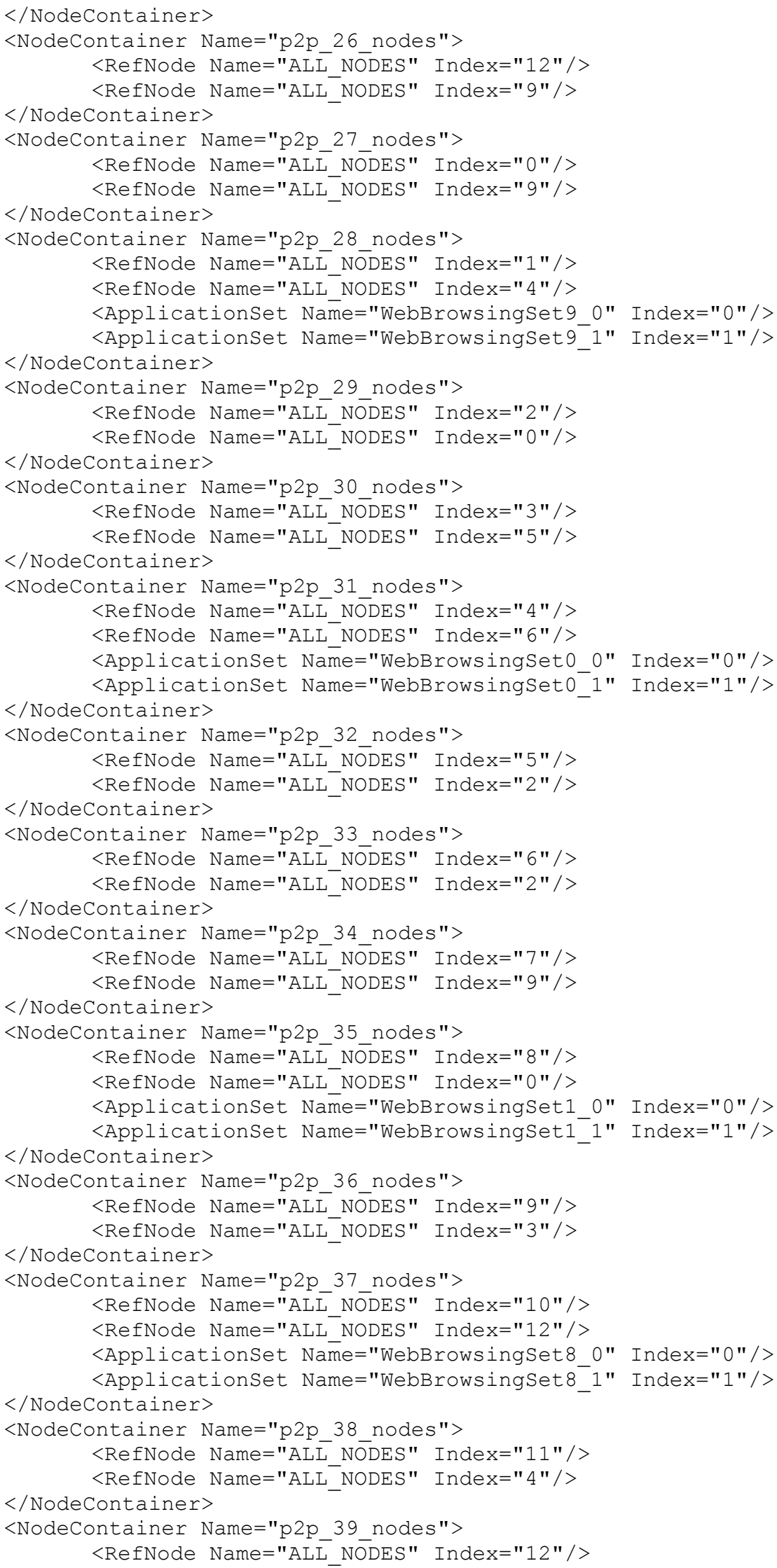


LLNL-TR-636200

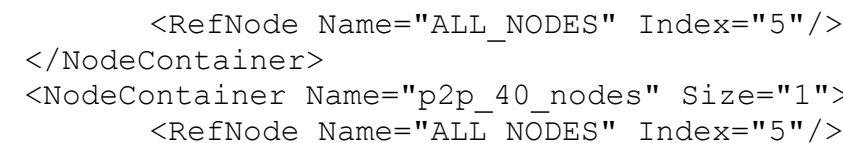


LLNL-TR-636200

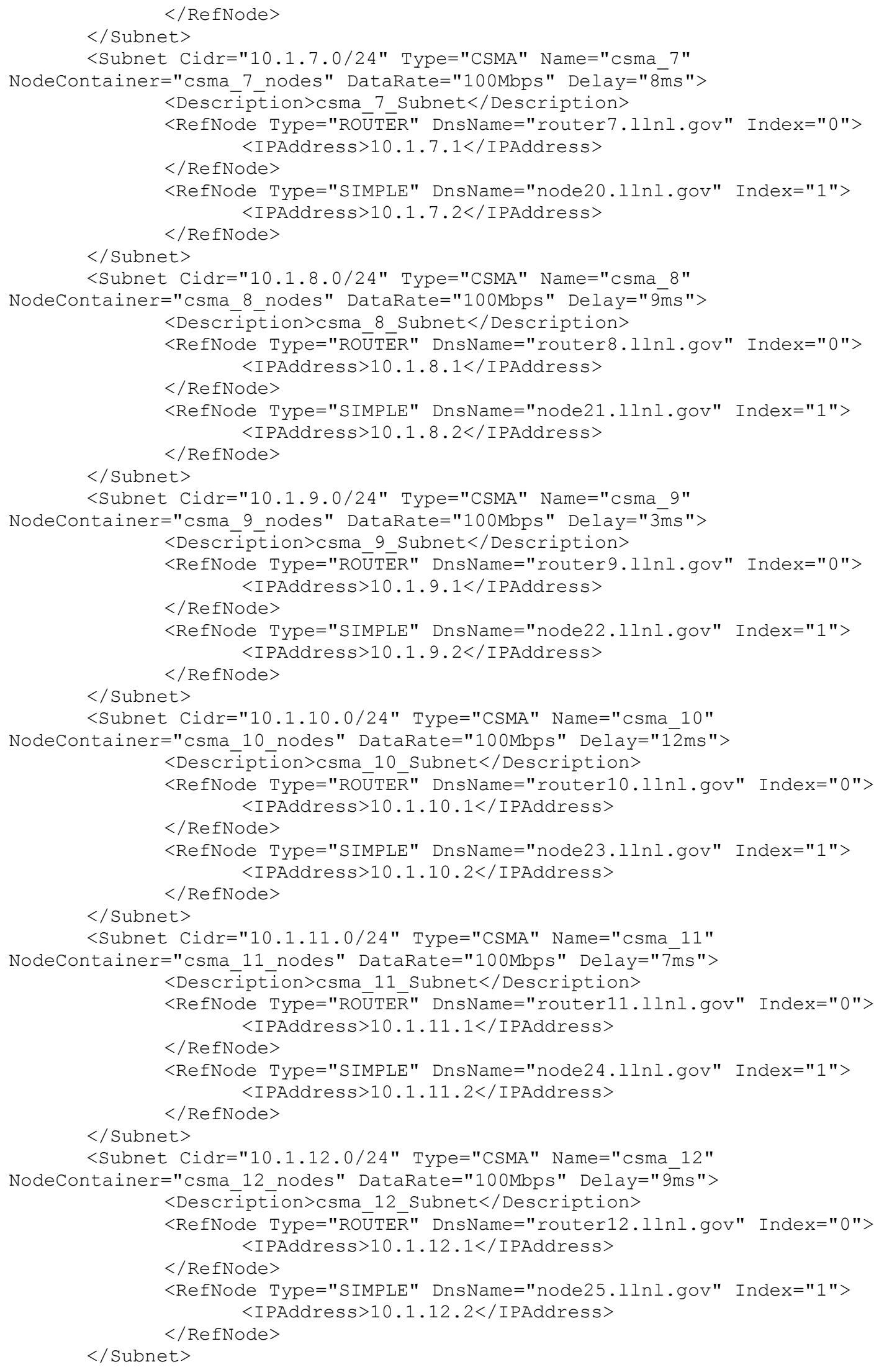


LLNL-TR-636200

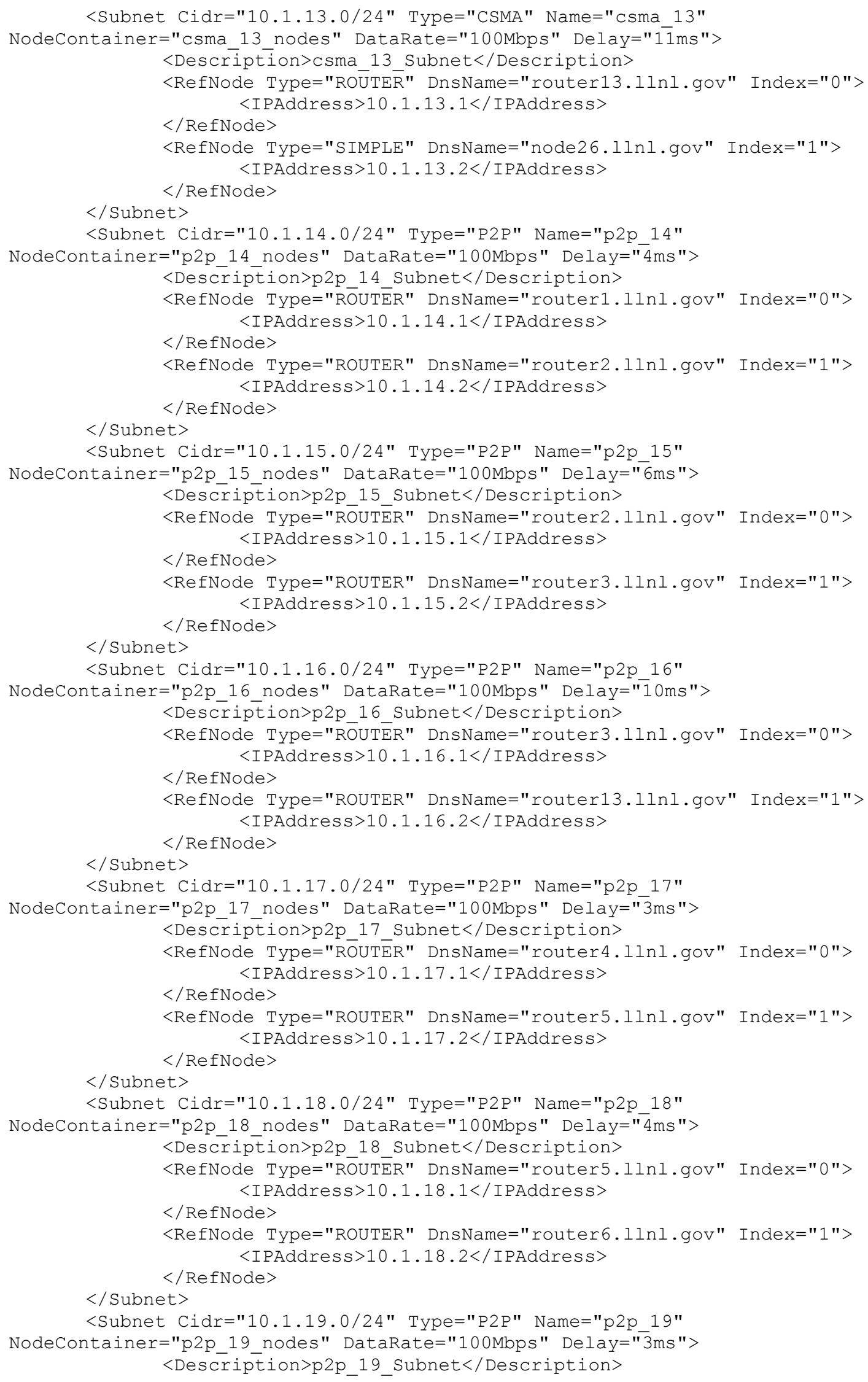


LLNL-TR-636200

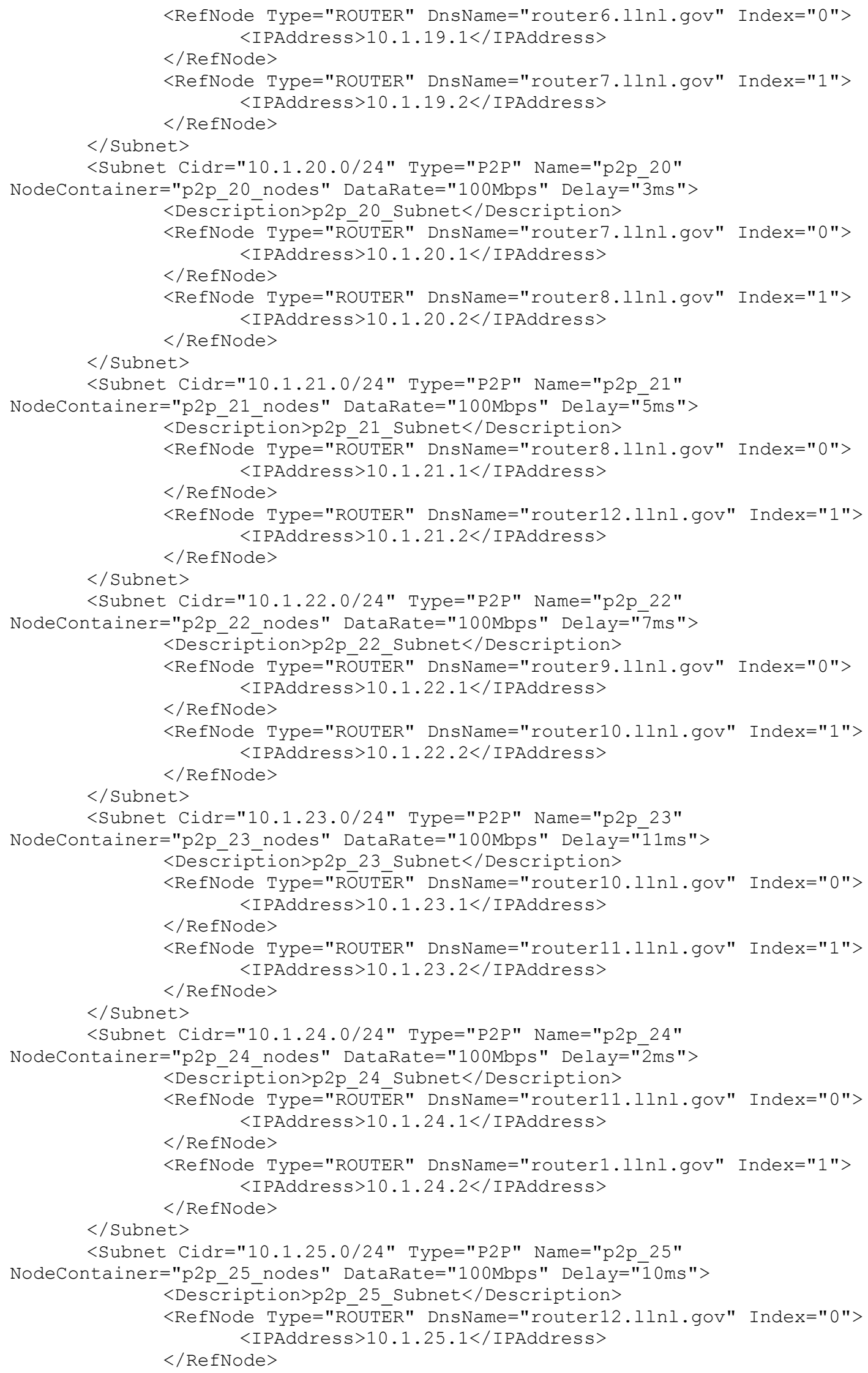


LLNL-TR-636200

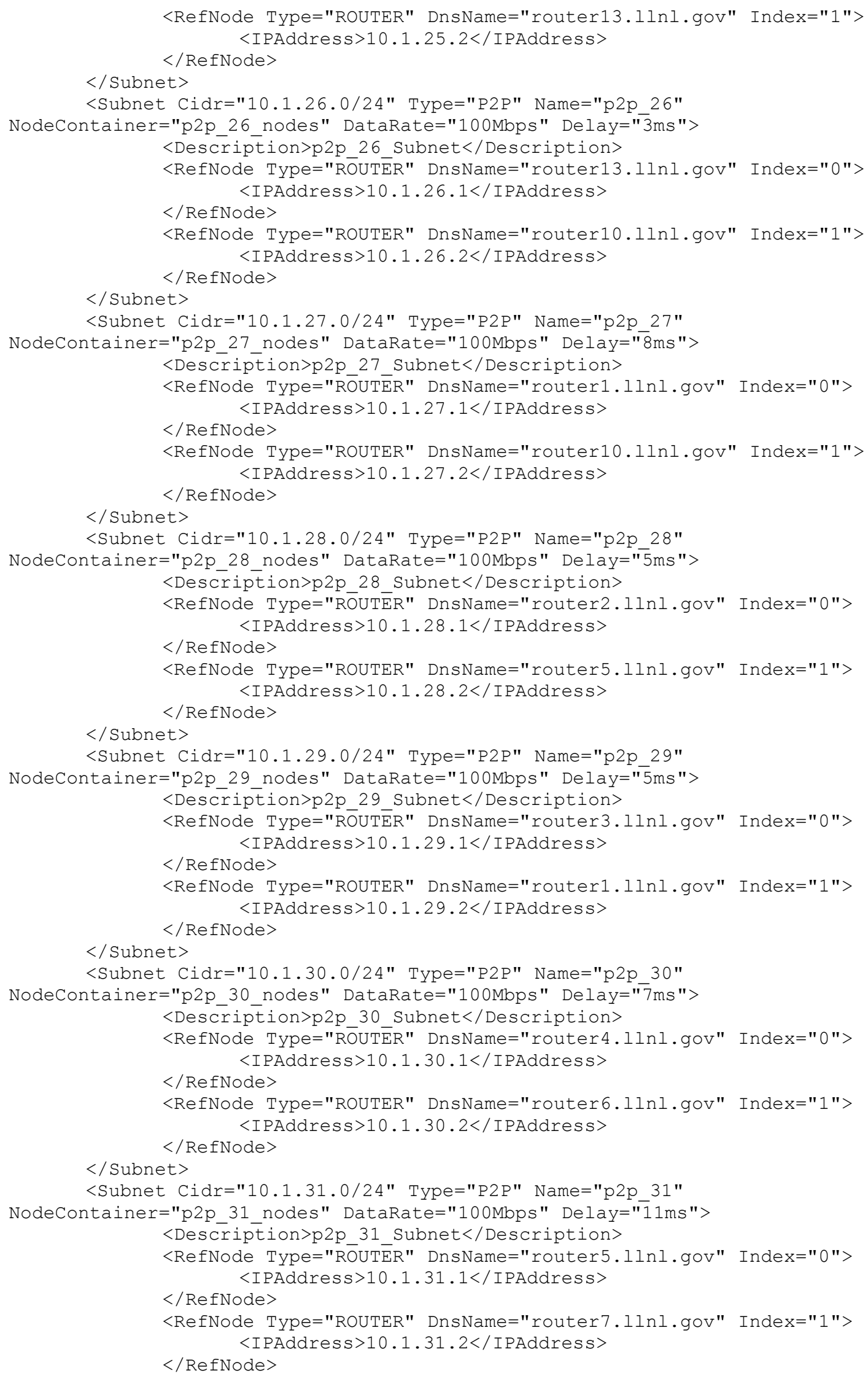


LLNL-TR-636200

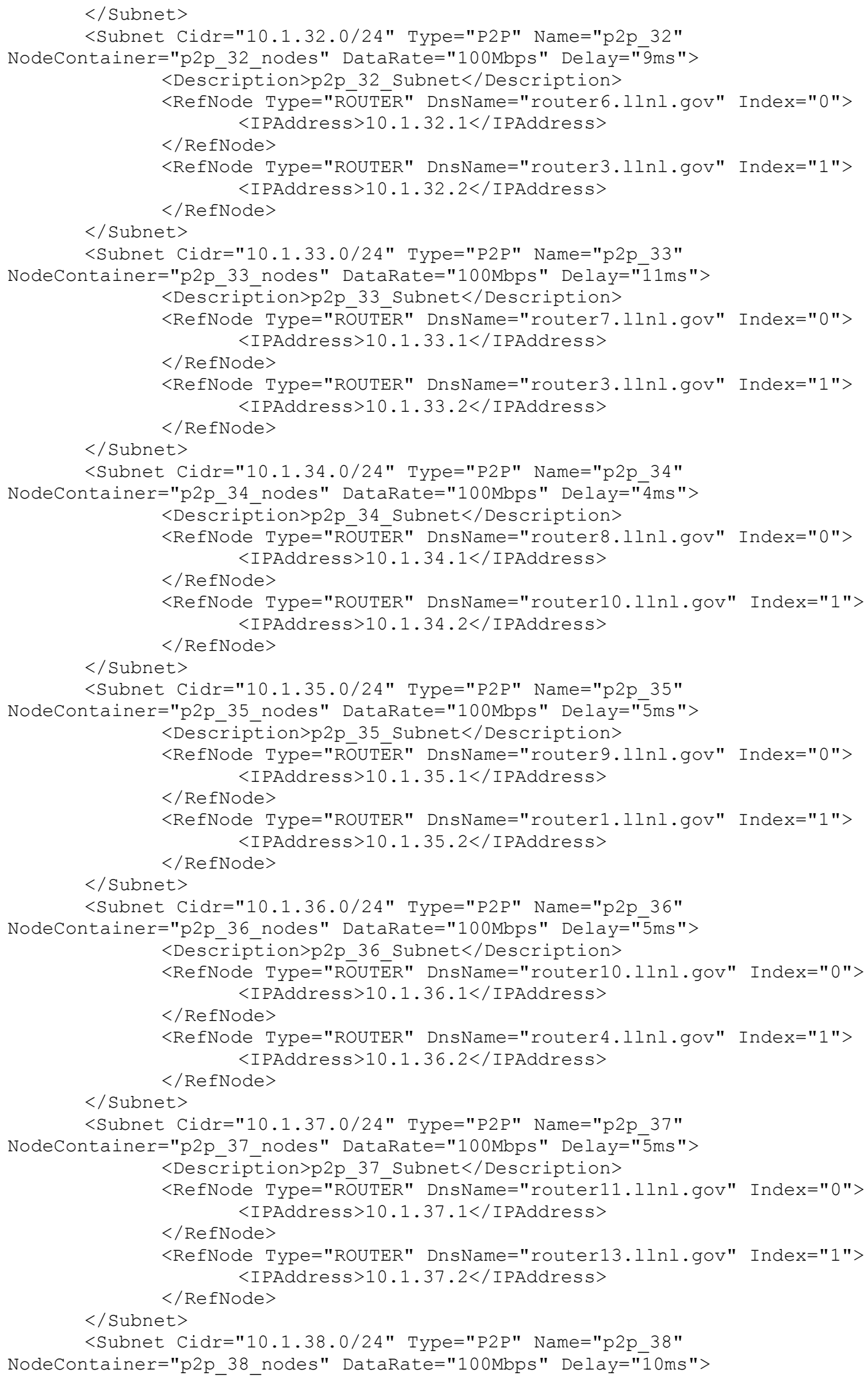


LLNL-TR-636200

$</$ Subnet $>$

$<$ Description $>$ p2p 38 Subnet $</$ Description $>$

$<$ RefNode Type="ROUTEER" DnsName="router12.1lnl.gov" Index="0"> <IPAddress $>10.1 .38 .1</$ IPAddress $>$

$</$ RefNode $>$

$<$ RefNode Type="ROUTER" DnsName="router5.1lnl.gov" Index="1"> $<$ IPAddress $>10.1 .38 .2</$ IPAddress $>$

$</$ RefNode $>$

<Subnet Cidr="10.1.39.0/24" Type="P2P" Name="p2p 39"

NodeContainer="p2p_39_nodes" DataRate="100Mbs" Delay="8ms" $>$ $<$ Description>p2p_39_Subnet</Description>

$<$ RefNode Type="RŌUTER" DnsName="router13.1lnl.gov" Index="0"> <IPAddress>10.1.39.1</IPAddress>

$</$ RefNode $>$

<RefNode Type="ROUTER" DnsName="router6.llnl.gov" Index="1"> $</$ RefNode $>$ $<$ IPAddress $>10.1 .39 .2</$ IPAddress $>$

$</$ Subnet $>$

<Subnet Cidr="10.1.40.0/24" Type="P2P" Name="p2p_40"

NodeContainer="p2p 40 nodes" DataRate="100Mbps" Delay="8ms"> $<$ Description>p2p_40_Subnet</Description>

$<$ RefNode Type="ROUTER" DnsName="router14.llnl.gov" Index="0"> <IPAddress $>10.1 .40 .1</$ IPAddress $>$

$</$ RefNode $>$

<RefNode Type="ROUTER" DnsName="router6.llnl.gov" Index="1"> $</$ RefNode $>$

<IPAddress $>10.1 .40 .2</$ IPAddress $>$

$</$ Subnet $>$

<Application xsi:type="PacketSinkAppType" Name="PacketSink"

Protocol="ns3::TcpSocketFactory" LocalAddress="0.0.0.0" LocalPort="80"

Start $=0.0 "$ Stop $=" 1000 " />$

$<$ Application xsi:type="OnOffAppType" Name="Client_0"

Protocol="ns3::TcpSocketFactory" DataRate="5000bps" PacketSize="500"

RemoteAddress="10.1.31.2" Port="80" Start="22.8263" Stop="1000"/>

<Application xsi:type="OnOffAppType" Name="Server_0"

Protocol="ns3::TcpSocketFactory" DataRate="5000bps" PacketSize="500"

RemoteAddress="10.1.31.1" Port="80" Start="22.8263" Stop="1000"/>

<Application xsi:type="OnOffAppType" Name="Client_1"

Protocol="ns3::TcpSocketFactory" DataRate="5000bps" Packetsize="500"

RemoteAddress="10.1.35.2" Port="80" Start="29.3714" Stop="1000"/>

<Application xsi:type="OnOffAppType" Name="Server_1"

Protocol="ns3::TcpSocketFactory" DataRate="5000bps" Packetsize="500"

RemoteAddress="10.1.35.1" Port="80" Start="29.3714" Stop="1000"/> <Application xsi:type="OnOffAppType" Name="Client_2"

Protocol="ns3::TcpSocketFactory" DataRate="5000bps" Packetsize="500"

RemoteAddress="10.1.13.2" Port="80" Start="63.0976" Stop="1000"/> <Application xsi:type="OnOffAppType" Name="Server_2"

Protocol="ns3::TcpSocketFactory" DataRate="5000bps" PacketSize="500"

RemoteAddress="10.1.13.1" Port="80" Start="63.0976" Stop="1000"/> <Application xsi:type="OnOffAppType" Name="Client_3"

Protocol="ns3::TcpSocketFactory" DataRate="5000bps" PacketSize="500"

RemoteAddress="10.1.4.2" Port="80" Start="9.21049" Stop="1000"/> <Application xsi:type="OnOffAppType" Name="Server 3"

Protocol="ns3::TcpSocketFactory" DataRate="5000bps" Packetsize="500"

RemoteAddress="10.1.4.1" Port="80" Start="9.21049" Stop="1000"/> <Application xsi:type="OnOffAppType" Name="Client_4"

Protocol="ns3: :TcpSocketFactory" DataRate="5000bps" PacketSize="500"

RemoteAddress="10.1.6.2" Port="80" Start="43.3701" Stop="1000"/> <Application xsi:type="OnOffAppType" Name="Server_4"

Protocol="ns3::TcpSocketFactory" DataRate="5000bps" Packetsize="500"

RemoteAddress="10.1.6.1" Port="80" Start="43.3701" Stop="1000"/> 
LLNL-TR-636200

<Application xsi:type="OnOffAppType" Name="Client_5" Protocol="ns3: :TcpSocketFactory" DataRate="5000bps" PacketSize="500" RemoteAddress="10.1.12.2" Port="80" Start="43.0863" Stop="1000"/> <Application xsi:type="OnOffAppType" Name="Server 5" Protocol="ns3::TcpSocketFactory" DataRate="5000bps" PacketSize="500" RemoteAddress="10.1.12.1" Port="80" Start="43.0863" Stop="1000"/> <Application xsi:type="OnOffAppType" Name="Client_6" Protocol="ns3::TcpSocketFactory" DataRate="5000bps" PacketSize="500" RemoteAddress="10.1.11.2" Port="80" Start="15.4082" Stop="1000"/> <Application xsi:type="OnOffAppType" Name="Server_6"

Protocol="ns3::TcpSocketFactory" DataRate="5000bps" PacketSize="500" RemoteAddress="10.1.11.1" Port="80" Start="15.4082" Stop="1000"/> <Application xsi:type="OnOffAppType" Name="Client_7" Protocol="ns3::TcpSocketFactory" DataRate="5000bps" PacketSize="500" RemoteAddress="10.1.14.2" Port="80" Start="7.70865" Stop="1000"/> <Application xsi:type="OnOffAppType" Name="Server_7" Protocol="ns 3: :TcpSocketFactory" DataRate="5000bps" PacketSize="500" RemoteAddress="10.1.14.1" Port="80" Start="7.70865" Stop="1000"/> <Application xsi:type="OnOffAppType" Name="Client_8" Protocol="ns3::TcpSocketFactory" DataRate="5000bps" PacketSize="500" RemoteAddress="10.1.37.2" Port="80" Start="88.9866" Stop="1000"/> <Application xsi:type="OnOffAppType" Name="Server_8" Protocol="ns3::TcpSocketFactory" DataRate="5000bps" Packetsize="500" RemoteAddress="10.1.37.1" Port="80" Start="88.9866" Stop="1000"/> <Application xsi:type="OnOffAppType" Name="Client_9" Protocol="ns3::TcpSocketFactory" DataRate="5000bps" PacketSize="500" RemoteAddress="10.1.28.2" Port="80" Start="89.3389" Stop="1000"/> <Application xsi:type="OnOffAppType" Name="Server_9"

Protocol="ns3: :TcpSocketFactory" DataRate="5000bps" PacketSize="500" RemoteAddress="10.1.28.1" Port="80" Start="89.3389" Stop="1000"/> <Application xsi:type="OnOffAppType" Name="Client 10"

Protocol="ns3::TcpSocketFactory" DataRate="5000bps" PacketSize="500" RemoteAddress="10.1.7.2" Port="80" Start="46.4268" Stop="1000"/> <Application xsi:type="OnOffAppType" Name="Server_10"

Protocol="ns3::TcpSocketFactory" DataRate="5000bps" PacketSize="500" RemoteAddress="10.1.7.1" Port="80" Start="46.4268" Stop="1000"/>

$<$ Applicationset Name="WebBrowsingSet0 0"> $<$ Application Name="PacketSink"T/> $<$ Application Name="Client_O"/>

$</$ Applicationset $>$

$<$ ApplicationSet Name="WebBrowsingSet0_1"> $<$ Application Name="PacketSink" $\overline{/>}$ $<$ Application Name="Server_0"/>

$</$ Applicationset $>$

$<$ Applicationset Name="WebBrowsingSet 1 0"> $<$ Application Name="PacketSink"/> $<$ Application Name="Client_1"/>

$</$ Applicationset $>$

$<$ ApplicationSet Name="WebBrowsingSet1_1"> $<$ Application Name="PacketSink" $\overline{/>}$ $<$ Application Name="Server_1"/>

$</$ ApplicationSet $>$

<Applicationset Name="WebBrowsingset2 0"> $<$ Application Name="PacketSink"/> $<$ Application Name="Client_2"/>

$</$ ApplicationSet $>$

$<$ ApplicationSet Name="WebBrowsingSet2_1"> $<$ Application Name="PacketSink" $\overline{/>}$ <Application Name="Server_2"/>

$</$ Applicationset $>$

$<$ Applicationset Name="WebBrowsingSet3_0"> $<$ Application Name="PacketSink"/> $<$ Application Name="Client_3"/> 
$</$ Applicationset $>$

$<$ ApplicationSet Name="WebBrowsingSet3 1"> $<$ Application Name="PacketSink" $\overline{/>}$ $<$ Application Name="Server_3"/>

$</$ ApplicationSet $>$

$<$ ApplicationSet Name="WebBrowsingSet 4 0"> $<$ Application Name="PacketSink"T> $<$ Application Name="Client_4"/>

$</$ ApplicationSet $>$

<Applicationset Name="WebBrowsingSet4_1"> $<$ Application Name="PacketSink"/> $<$ Application Name="Server_4"/>

$</$ Applicationset $>$

<ApplicationSet Name="WebBrowsingSet5 0"> $<$ Application Name="PacketSink" $/>$ $<$ Application Name="Client_5"/>

$</$ Applicationset $>$

$<$ Applicationset Name="WebBrowsingSet5_1"> <Application Name="PacketSink"/> $<$ Application Name="Server_5"/>

$</$ Applicationset $>$

<ApplicationSet Name="WebBrowsingSet 6 0"> $<$ Application Name="PacketSink" /> $<$ Application Name="Client_6"/>

$</$ Applicationset $>$

<ApplicationSet Name="WebBrowsingSet 6 1"> $<$ Application Name="PacketSink"T/> $<$ Application Name="Server_6"/>

$</$ Applicationset $>$

$<$ ApplicationSet Name="WebBrowsingSet7_0"> $<$ Application Name="PacketSink" $\overline{/}>$ $<$ Application Name="Client_7"/>

$</$ ApplicationSet $>$

$<$ Applicationset Name="WebBrowsingSet 7 1"> $<$ Application Name="PacketSink" $\overline{>}>$ $<$ Application Name="Server_7"/>

$</$ ApplicationSet $>$

<ApplicationSet Name="WebBrowsingSet8_0"> $<$ Application Name="PacketSink" $/>$ $<$ Application Name="Client_8"/>

$</$ ApplicationSet $>$

$<$ ApplicationSet Name="WebBrowsingSet 8 1"> <Application Name="PacketSink"/> $<$ Application Name="Server_8"/>

$</$ ApplicationSet $>$

$<$ ApplicationSet Name="WebBrowsingSet9_0"> $<$ Application Name="PacketSink" $\overline{/>}$ $<$ Application Name="Client_9"/>

$</$ Applicationset $>$

$<$ ApplicationSet Name="WebBrowsingSet 9 1"> $<$ Application Name="PacketSink"T/> $<$ Application Name="Server_9"/>

$</$ ApplicationSet $>$

$<$ Applicationset Name="WebBrowsingSet10_0"> $<$ Application Name="PacketSink"/> $<$ Application Name="Client_10"/>

$</$ Applicationset $>$

$<$ Applicationset Name="WebBrowsingSet10 1"> $<$ Application Name="PacketSink"/> $<$ Application Name="Server_10"/>

$</$ NetSim $>$

$</$ Applicationset $>$ 
LLNL-TR-636200

\section{Appendix B. AddSystemld Python script}

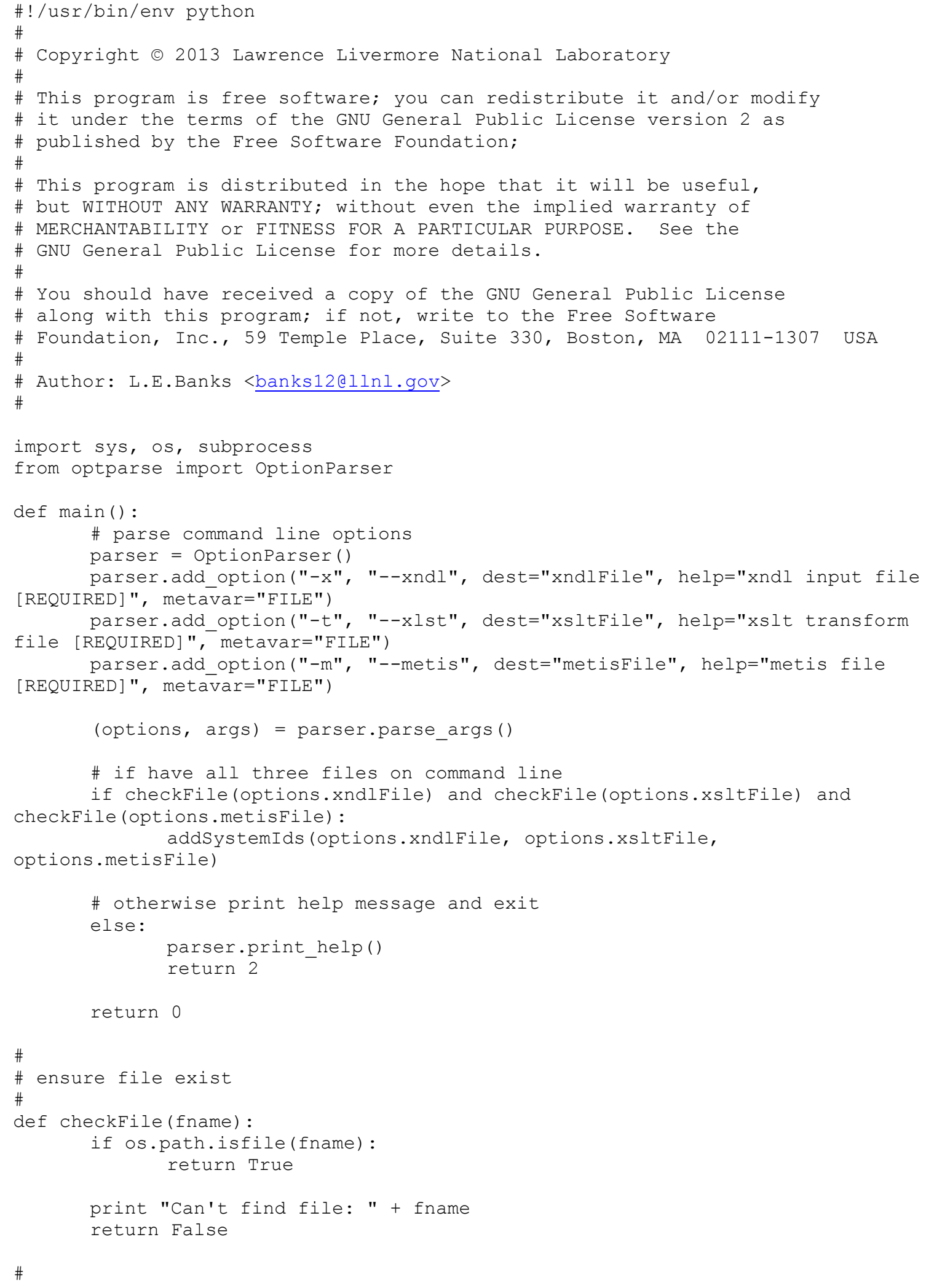




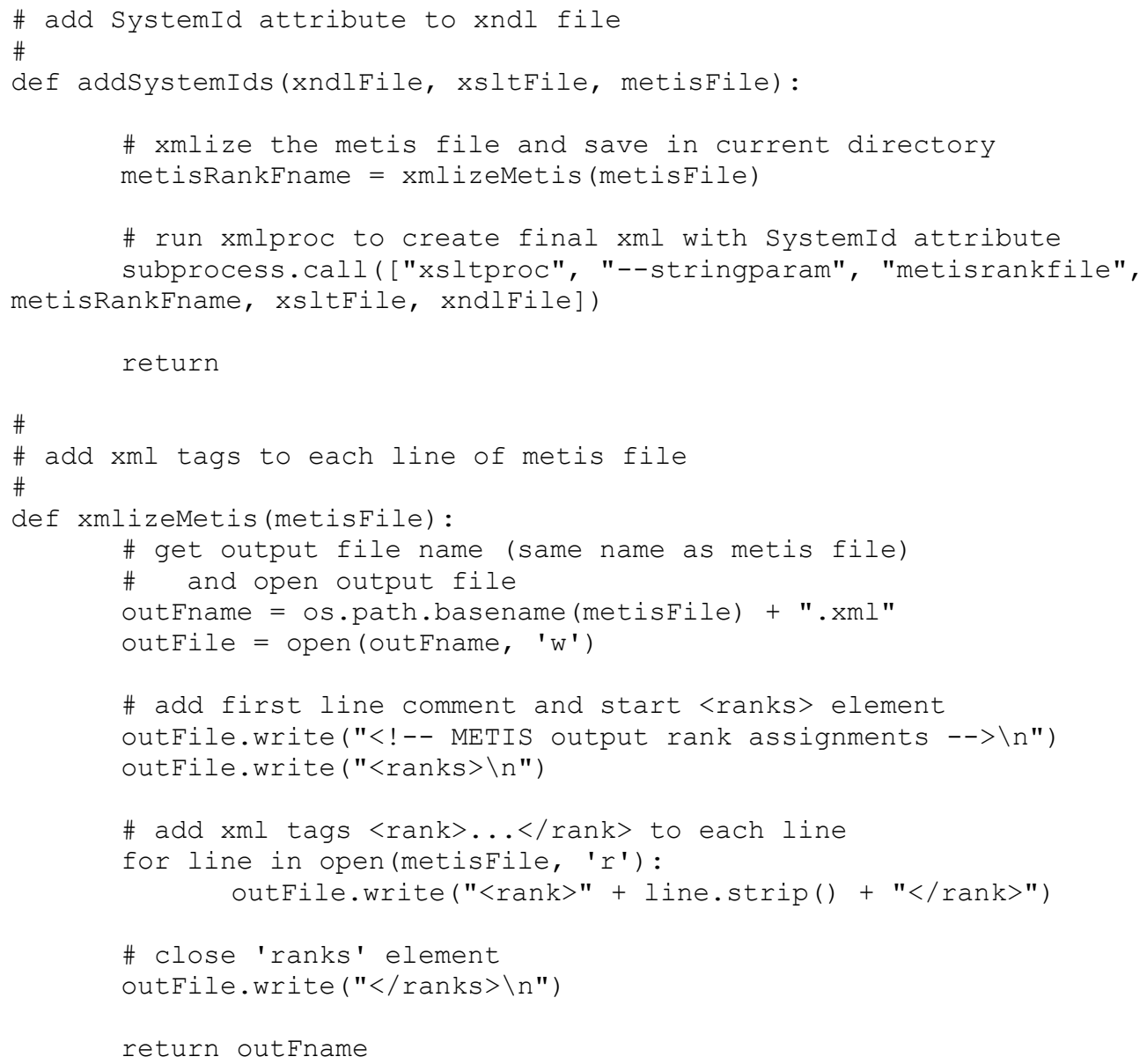

